\title{
Interpretable Machine Learning of Voluminous Scattering data E. $\mathrm{Kim}^{1}$ \\ ${ }^{1}$ Cornell University \\ ek436@cornell.edu
}

Decades of efforts in improving instrumentation and sensors led us to the age of voluminous scattering data. However, the radically increased volume and experimental control present new challenges. I will discuss how these challenges can be embraced and turned into opportunities by employing machine learning. The rigorous framework for scientific understanding physicists enjoy through our celebrated tradition requires the interpretability of any machine learning essential. I will discuss our recent results using machine learning approaches designed to be interpretable from the outset. Specifically, I will present discovering order parameters and its fluctuations in voluminous X-ray diffraction data using a X-ray TEmperatures Clustering (X-TEC) we introduced in Ref[1].

[1] Venderley et al, https://arxiv.org/abs/2008.03275. 